\title{
Erratum to: Experiences and attitudes of residents regarding a community-based genome cohort study in Japan: a population-based, cross-sectional study
}

Keiko Miyamoto ${ }^{1 *}$, Miho Iwakuma $^{1}$ and Takeo Nakayama ${ }^{2}$

\section{Erratum}

After the publication of this article the authors noticed that an incorrect version of Table 3 is shown. The correct version can be seen on the following page.

\footnotetext{
Author details

'Department of Medical Communication, Kyoto University School of Public Health, Yoshidakonoe-cho, Sakyo-ku, Kyoto \#6068501, Japan. ${ }^{2}$ Department of Health Infomatics, Kyoto University School of Public Health, Yoshidakonoe-cho, Sakyo-ku, Kyoto \#6068501, Japan.
}

Received: 29 November 2016 Accepted: 8 December 2016 Published online: 12 January 2017

\section{Reference}

1. Keiko M, Miho I, Takeo N. Experiences and attitudes of residents regarding a community-based genome cohort study in Japan: a population-based cross-sectional study. BMC Med Genomics. 2016;9:14. doi:10.1186/s12920016-0175-8.

\footnotetext{
* Correspondence: miyamoto.keiko.87w@st.kyoto-u.ac.jp

${ }^{1}$ Department of Medical Communication, Kyoto University School of Public Health, Yoshidakonoe-cho, Sakyo-ku, Kyoto \#6068501, Japan
} 


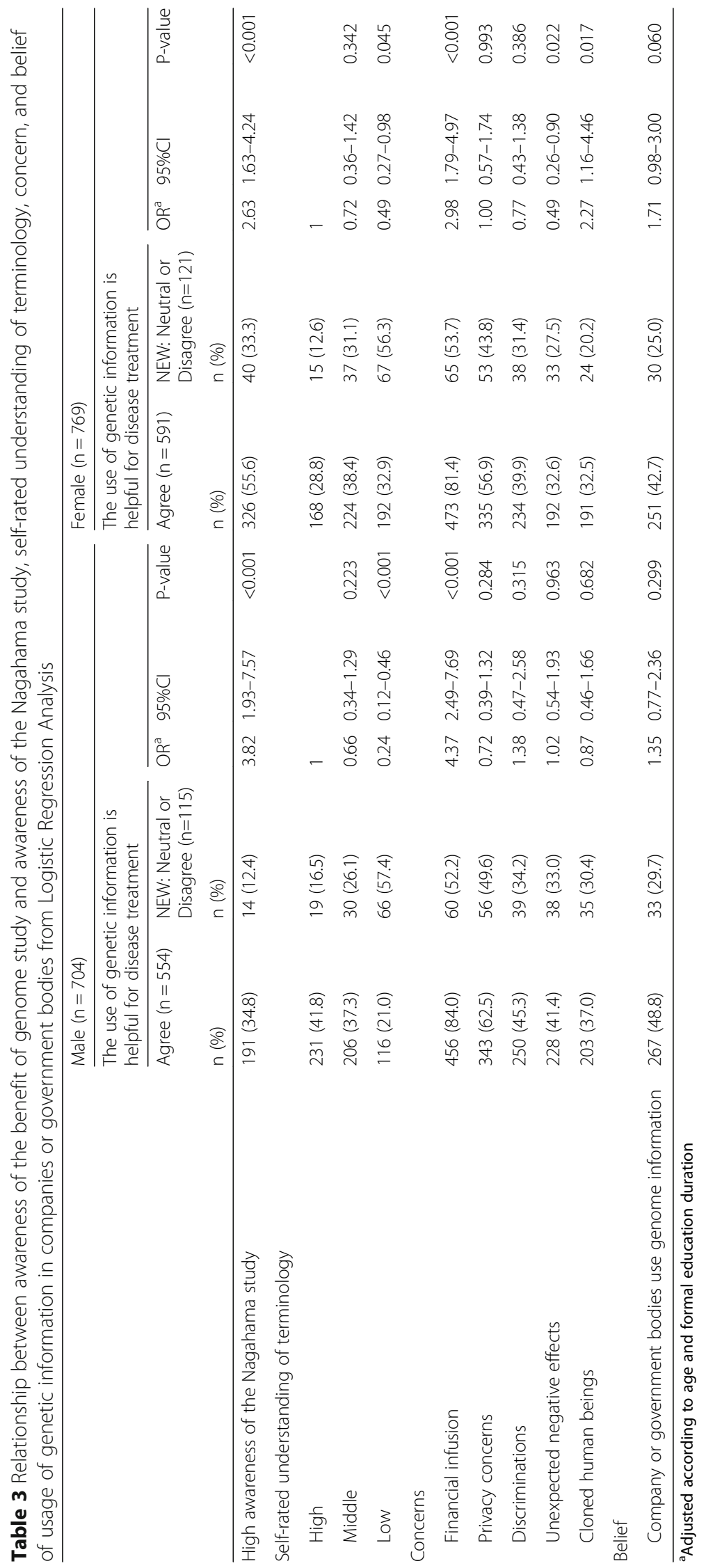

